\title{
Análisis de la satisfacción en el servicio de salud desde la perspectiva de usuarios y personal de contacto (c) (1) \&() (2)
}

\author{
Analysis of satisfaction in the health service from the perspective of users and \\ contact staff
}

Denise Liliana Pazmiño Garzón. ${ }^{1}$, Zoe Rodríguez Cotilla. ${ }^{2}$, Marco Vinicio Carrión Torres. ${ }^{3}$ \& Yolanda Patricia Moncayo Sánchez. ${ }^{4}$

Recibido: 29-05-2019 / Revisado: 20-06-2019 /Aceptado: 30-07-2019/ Publicado: 06-09-2019

\begin{abstract}
.
DOI: https://doi.org/10.33262/cienciadigital.v3i3.3.775

The general objective of this work is to analyze the determinant factors and dimensions of satisfaction in the health service of the CASI (Integral Health Care Center) from the perspective of users (students, employees, teachers and managers) and service providers. To achieve this objective, two parallel investigations have been carried out through the application of user and contact personnel surveys, carrying out a comparative analysis of the factors considered based on the assessment of their importance and satisfaction, identifying discrepancies between the assessments granted by Study groups on key satisfaction factors. The analysis also made it possible to define and characterize different types of users with respect to their satisfaction assessment. The identified deficiencies involve several factors such as professionalism, courtesy of contact personnel, as a fundamental element in the satisfaction of the patient identified as a user of the service, as well as the cleanliness of the facilities, ease of access to the service, speed in the service identified as the response capacity, one of the five dimensions of service quality. The factors that registered lower than average assessments constitute gaps in the quality of the service, reflected in areas of improvement in the process of providing the health service tending to increase the levels of user satisfaction.
\end{abstract}

Keywords: Health service, satisfaction, perception, users, contact staff.

\footnotetext{
${ }^{1}$ Escuela Superior Politécnica de Chimborazo, Facultad de Administración de Empresas. Riobamba, Ecuador. denise.pazmino@espoch.edu.ec

${ }^{2}$ Universidad de La Habana. Facultad de Economía. Profesora titular. La Habana,Cuba. zoe@ @ec.uh.cu

${ }^{3}$ Universidad de las Fuerzas Armadas, Riobamba, Ecuador. mvct1980@ hotmail.com

${ }^{4}$ Escuela Superior Politécnica de Chimborazo, Facultad de Administración de Empresas Riobamba, Ecuador. yolanda.moncayo@espoch.edu.ec
} 


\section{Resumen}

El objetivo general del presente trabajo es analizar los factores y dimensiones determinantes de la satisfacción en el servicio de salud del CASI (Centro de Atención de Salud Integral) desde la perspectiva de los usuarios (estudiantes, empleados, docentes y directivos) y prestadores del servicio. Para alcanzar este objetivo se han desarrollado dos investigaciones paralelas mediante la aplicación de encuestas a usuarios y personal de contacto, realizando un análisis comparativo de los factores considerados a partir de la valoración de importancia y satisfacción de los mismos, identificando discrepancias entre las valoraciones concedidas por los grupos de estudio sobre factores clave de la satisfacción. El análisis posibilitó además definir y caracterizar diferentes tipos de usuarios con respecto a su evaluación de la satisfacción. Las deficiencias identificadas involucran varios factores tales como el profesionalismo, cortesía del personal de contacto, como elemento fundamental en la satisfacción del paciente identificado como usuario del servicio, así como también la limpieza de las instalaciones, facilidad en el acceso al servicio, rapidez en el servicio identificada como la capacidad de respuesta, una de las cinco dimensiones de la calidad del servicio. Los factores que registraron valoraciones inferiores a la media constituyen brechas en la calidad del servicio, reflejadas en áreas de mejora en el proceso de prestación del servicio de salud tendientes a incrementar los niveles de satisfacción de los usuarios.

Palabras claves: Servicio de salud, satisfacción, percepción, usuarios, personal de contacto.

\section{Introducción}

La preocupación por la calidad en la asistencia sanitaria es tan antigua como el propio ejercicio médico. Su origen se encuentra en Papiros egipcios, en el Código de Hammurabi o en el tratado La Ley del propio Hipócrates. En todos los casos, buscar lo mejor para el paciente, identificado como usuario del servicio de salud, es la esencia del ejercicio, apareciendo el inseparable binomio ética y calidad.

A continuación, se presentan los aportes de los autores más representativos en torno a calidad del servicio, percepción y satisfacción:

Es así como Carlzon (1987) expresa que la calidad en los servicios es la acumulación de experiencias satisfactorias del cliente que inicia a partir de los primeros 30 segundos de contacto y culminan en los 30 segundos finales conocidos como "momentos de verdad".

Con este análisis, se precisa que la calidad del servicio no sólo considera el contenido brindado, sino también la interacción psicológica que tiene lugar en la transacción.

Otros autores como Parasuraman, A.; Zeithmal, V. y Berry, L. (1993) definen a la calidad en el servicio como "La amplitud de la discrepancia o diferencia que exista entre las expectativas o deseos de los clientes y sus percepciones" y la refiere desde su modelo SERVQUAL basado en cinco dimensiones, como lo expresa (Castillo, 2005):

- Elementos tangibles: relacionada con la apariencia de las instalaciones físicas, personal y material de comunicación.

- Confiabilidad: entendida como la habilidad de desarrollar el servicio prometido precisamente como se pactó y con exactitud. 
- Capacidad de respuesta: concebida como la buena voluntad de ayudar a sus clientes y brindar un servicio rápido.

- Seguridad: referida al conocimiento de los empleados sobre lo que hacen, su cortesía y su capacidad de transmitir confianza.

- Empatía: considerada como la capacidad de brindar cuidado y atención personalizada a sus clientes.

El objetivo fundamental que persiguen las organizaciones es la satisfacción integral del cliente, es por tal razón que el autor Thompson (2005) contempla tres requerimientos que se basan en:

- El rendimiento percibido: referido al desempeño (en cuanto a la entrega de valor) que el cliente considera haber obtenido luego de adquirir un producto o servicio. Siendo este el "resultado" que el cliente "percibe" al momento de adquirir el producto o servicio. Las características de esta percepción son determinadas desde el punto de vista del cliente, no de la organización; de los resultados obtenidos; de las percepciones del cliente, no necesariamente de la realidad. Además, los criterios externos de otras personas, el estado de ánimo, sus experiencias previas y su razonamiento, influyen en el impacto. Es decir, "el rendimiento percibido", inicia y finaliza en el "cliente".

- Las expectativas: denominadas como "esperanzas" que los clientes tienen por conseguir algo. Se producen por varias situaciones: promesas en la oferta de las organizaciones o de sus competidores, experiencias anteriores de compra, y/u opiniones de personas cercanas. Es de vital importancia monitorear paulatinamente el nivel de expectativas de los clientes, como planeación estratégica.

- Los niveles de satisfacción: Luego de adquirir o consumir/usar un producto o servicio, los clientes experimentan tres niveles de satisfacción:

a) Insatisfacción: cuando el rendimiento no supera la expectativa del cliente;

b) Satisfacción: cuando el rendimiento coincide con la expectativa del cliente; y,

c) Complacencia: cuando el rendimiento excede la expectativa del cliente.

Criterio que fue complementado por Gallego (2002), quien define la calidad del servicio mediante la denominada "Ecuación de la calidad", identificando a la satisfacción del cliente en tres grados, que están en correspondencia con la fidelidad del cliente.

- Percepción $<$ Expectativa $=$ Cliente insatisfecho $=$ Mala calidad. El cliente no vuelve

- Percepción $=$ Expectativa $=$ Cliente satisfecho $=$ Buena calidad . El cliente puede elegir otros establecimientos

- Percepción $>$ Expectativa $=$ Cliente encantado $=$ Excelente calidad. El cliente es fiel . Según la ISO 9000:2015 la calidad de los productos y servicios de una organización está determinada por la capacidad para satisfacer a los clientes y por el impacto previsto sobre las partes interesadas pertinentes. La calidad de los productos y servicios incluye no sólo su función y desempeño, sino también su valor percibido y el beneficio para el cliente.

En el ámbito de las percepciones, las actitudes previas a la compra de los consumidores condicionan su percepción post-uso de servicio. Los consumidores con expectativas inicialmente favorables tienden a estar satisfechos, aún en el caso de que la experiencia sea negativa y viceversa, si las expectativas son poco favorables, la experiencia será probablemente percibida como insatisfactoria (Rust \& Oliver, 1994) 
Las percepciones del cliente sobre la calidad del servicio pueden ser inestables en el tiempo. La presencia de elementos subjetivos en la formación del juicio del cliente puede conducir a percepciones variables sobre la calidad de un mismo servicio, prestado del mismo modo, en diferentes momentos de tiempo (Curry, 2002)

En lo que respecta a la satisfacción del paciente, ésta ha sido considerada tradicionalmente como factor determinante de la futura utilización de los servicios de salud, o bien consecuencia de la utilización de estos. La conceptualización predominante es la de Koos (1954) y Avedis Donabedian (1966), para quienes la satisfacción del paciente es una medida del resultado de la interacción entre los profesionales de la salud y el paciente.

La repercusión de la satisfacción del paciente en la calidad de la atención médica ha sido centro de debate en los espacios educativos y asistenciales; el más difundido y aceptado el propuesto por Avedis Donabedian en 1966. Para este autor, la satisfacción del paciente en relación con los servicios de salud es un punto nodal para determinar la calidad de la atención médica que se presta. Donabedian entiende la satisfacción del paciente, como una medida de eficacia e incluso de control objetivo de la calidad de los servicios de salud.

Respecto al personal de contacto, éste tiene una fuerte influencia sobre la satisfacción del cliente. Sobre su importancia, Sainz de Vicuña (2001) recalca su influencia sobre la imagen y las actitudes del usuario hacia la organización.

\section{Metodología}

En relación al objetivo planteado se definen las siguientes hipótesis de investigación:

H1: Los elementos del servicio con mayor importancia desde la perspectiva de los prestadores del servicio coinciden con los atributos más satisfactorios para los usuarios.

H2: Las dimensiones de la satisfacción del usuario difieren de las consideradas por los prestadores del servicio.

H3: Existen grupos de usuarios diferenciados según la forma en que evalúan la satisfacción con el servicio de salud.

Para dar cumplimiento al objetivo planteado, la investigación se desarrolló utilizando el enfoque cuantitativo a través de la recolección de datos mediante muestras y encuestas estructuradas, con base en la medición numérica y el análisis estadístico, para establecer patrones de comportamiento y comprobar teorías (Hernández, et al.; 2010; Duane, 2001; Del Cid, et al., 2007). Además, se aplica el enfoque cualitativo, pues al considerar que la satisfacción del cliente se fundamenta en las percepciones del mismo, este enfoque es ideal para la obtención de este tipo de información.

Se trabajó con dos grupos de población; el primero integrado por el personal que labora en el CASI, el segundo conformado por los miembros de la comunidad politécnica (estudiantes, empleados, docentes y directivos), usuarios del servicio. En el primer caso se aplica un censo por tratarse de una población reducida (11 personas). Para el cálculo de la muestra, en la segunda población se aplica la fórmula para poblaciones finitas y se utiliza el muestreo proporcional al tamaño del estrato (Del Cid, et al. 2007, Hernández, et al., 2010).

Para la aplicación de la técnica de la encuesta, fue necesario elaborar dos tipos de cuestionarios a utilizar, tanto para clientes internos, así como para los clientes externos. Los instrumentos contemplan variables consideradas en la gestión de la calidad de servicios que permitan identificar debilidades y fortalezas en los procesos efectuados en el CASI. En la encuesta dirigida a clientes internos, la medición de las variables se realiza mediante una 
ISSN: 2602-8085

escala de Likert de cinco posiciones que oscilaba desde "mucho peor de lo que esperaba" hasta "mucho mejor de lo que esperaba", reflejando así la modelización de la satisfacción según el paradigma disconfirmatorio subjetivo. En la encuesta a clientes internos la medición de las variables se realiza con una escala tipo Likert de cinco posiciones que oscilaban desde "sin importancia" hasta "altamente importante", con objeto de evaluar la relevancia concedida por los prestadores del servicio a los atributos utilizados por los usuarios en los juicios de satisfacción.

La validez de los cuestionarios, se comprobó mediante el cálculo del coeficiente de fiabilidad denominado Alfa de Cronbach, el cual "se establece como un índice de factor para evaluar el grado en que los ítems de un instrumento están correlacionados" (González y Pazmiño, 2015:64).

\section{Resultados}

Hipótesis 1. Discrepancias en los juicios de satisfacción entre prestadores del servicio y usuarios

Para comprobar la primera hipótesis se obtienen las puntuaciones medias de los diferentes factores de satisfacción tanto para los usuarios como para los prestadores del servicio. Posteriormente, se clasifican en dos categorías según su valor promedio sea superior o inferior a la media global de los factores de satisfacción y se comparan los resultados obtenidos del estudio de los usuarios con los obtenidos en el estudio de los prestadores del servicio. (Tabla 1).

Tabla 1. Comparación de los determinantes de la satisfacción entre usuarios y prestadores del servicio

\begin{tabular}{|c|c|c|c|c|}
\hline \multirow{2}{*}{$\begin{array}{c}\text { Factores ordenados de forma } \\
\text { descendente por el grado } \\
\text { de importancia para los prestadores } \\
\text { del servicio }\end{array}$} & \multicolumn{2}{|c|}{$\begin{array}{c}\text { Importancia } \\
\text { Prestadores del servicio }\end{array}$} & \multicolumn{2}{|c|}{ Satisfacción usuarios } \\
\hline & Media & Nivel & Media & Nivel \\
\hline \multicolumn{5}{|c|}{ Factores con niveles de importancia superiores a la media } \\
\hline Limpieza de las instalaciones & 5,00 & Superior & 3,66 & Inferior \\
\hline Cortesía del personal & 4,91 & Superior & 3,53 & Inferior \\
\hline Profesionalismo & 4,91 & Superior & 3,73 & Inferior \\
\hline Claridad de la información & 4,91 & Superior & 4,11 & Superior \\
\hline Cumplimiento del horario de servicio & 4,82 & Superior & 4,85 & Superior \\
\hline Variedad en servicios & 4,82 & Superior & 3,59 & Inferior \\
\hline \multicolumn{5}{|c|}{ Factores con niveles de importancia inferiores a la media } \\
\hline Atención personalizada & 4,73 & Inferior & 3,54 & Inferior \\
\hline Imagen y prestigio institucional & 4,73 & Inferior & 4,03 & Superior \\
\hline Facilidad en el acceso al servicio & 4,64 & Inferior & 3,64 & Inferior \\
\hline Rapidez en el servicio & 4,55 & Inferior & 3,59 & Inferior \\
\hline Instalaciones físicas & 4,55 & Inferior & 3,70 & Inferior \\
\hline Apariencia del personal de la entidad & 4,55 & Inferior & 3,72 & Inferior \\
\hline Media & 4,76 & - & 3,81 & - \\
\hline
\end{tabular}

Fuente: SPSS versión 22 
La comparación de los resultados obtenidos a partir de la investigación a los dos estratos evidencia en términos generales, la no coincidencia entre los factores con relevancia superior a la media para los prestadores del servicio y los niveles de satisfacción de los usuarios.

En forma precisa, la coincidencia de valoraciones se observa únicamente en dos de los doce factores evaluados, que presentan niveles de importancia para los prestadores del servicio superiores a la media. En el conjunto de factores con relevancia inferior, se presenta un mayor número de discrepancias, dado que sólo coinciden las valoraciones en siete de los doce factores analizados.

Los hallazgos confirman en términos generales la primera hipótesis y revelan además que los aspectos concernientes a la entrega del servicio sanitario por parte del personal de contacto, si bien son relevantes para los usuarios, deben ser mejorados para incrementar los niveles de satisfacción de los mismos.

H2: Las dimensiones de la satisfacción del usuario difieren de las consideradas por los prestadores del servicio.

Con la finalidad de identificar las dimensiones de la satisfacción desde la perspectiva de los usuarios y prestadores del servicio se procedió a analizar los resultados obtenidos del nivel de importancia concedido por los grupos de estudio a cada atributo considerado, visualizados a continuación:

Tabla 2. Comparación de las valoraciones de importancia otorgada a los factores considerados entre usuarios y personal de contacto

\begin{tabular}{|l|c|c|c|}
\hline $\begin{array}{c}\text { Factores ordenados de forma } \\
\text { descendente por el grado } \\
\text { de importancia para los prestadores } \\
\text { del servicio }\end{array}$ & $\begin{array}{c}\text { Importancia } \\
\text { Prestadores } \\
\text { del servicio }\end{array}$ & $\begin{array}{c}\text { Importancia } \\
\text { Usuarios }\end{array}$ & Brecha \\
\cline { 2 - 4 } & Media & Media & - \\
\hline Limpieza de las instalaciones & 5,00 & 5,00 & 0,00 \\
\hline Cortesía del personal & 4,35 & 4,76 & 0,41 \\
\hline Profesionalismo & 4,91 & 5,00 & 0,09 \\
\hline Claridad de la información & 4,91 & 4,29 & $-0,62$ \\
\hline Cumplimiento del horario de servicio & 4,61 & 5,00 & 0,39 \\
\hline Variedad en servicios & 4,14 & 4,49 & 0,35 \\
\hline Atención personalizada & 4,73 & 4,22 & $-0,51$ \\
\hline Imagen y prestigio institucional & 4,33 & 4,40 & 0,07 \\
\hline Facilidad en el acceso al servicio & 4,64 & 4,94 & 0,30 \\
\hline Rapidez en el servicio & 4,55 & 4,77 & 0,22 \\
\hline Instalaciones físicas & 4,55 & 4,62 & 0,07 \\
\hline Apariencia del personal de la entidad & 4,55 & 4,21 & $-0,34$ \\
\hline
\end{tabular}

Fuente: SPSS versión 22 
Los resultados de este análisis determinan las brechas en el nivel de importancia concedida por parte de los estratos de estudio a los factores evaluados, es así que a cuatro de doce atributos los usuarios otorgan mayor importancia, mientras que en siete atributos los prestadores del servicio confieren mayor nivel de importancia en la prestación del servicio sanitario. Se presenta además una coincidencia en la valoración del atributo limpieza de las instalaciones, tanto usuarios como prestadores del servicio le han asignado el mayor nivel de importancia.

H3: Existen grupos de usuarios diferenciados según la forma en que evalúan la importancia y satisfacción con el servicio de salud.

La intensidad de la relación entre variables puede ser medida a través de medidas de asociación, de concordancia o de correlación.

El nivel de concordancia medido con el coeficiente Kappa se interpreta así:

- Para valores iguales o mayores que 0.75 de Kappa de Cohen existe una excelente posición de concordancia entre las dos variables (Importancia y satisfacción).

- Para valores entre 0.40 y 0.75 de Kappa de Cohen existe una concordancia de suficiente a buena entre las dos variables (Importancia y satisfacción).

- Para valores menores de 0.40 de Kappa de Cohen existe una débil concordancia entre las dos variables (Importancia y satisfacción).

El nivel de significación asociado al valor de Kappa habla del rechazo en la hipótesis de falta de concordancia entre las dos variables, de tal manera que valores de probabilidad menores que 5\% llevan al rechazo de la hipótesis de falta de concordancia.

Tabla 3. Nivel de concordancia entre Importancia y Satisfacción

\begin{tabular}{|c|c|c|c|c|c|c|c|c|}
\hline \multirow{2}{*}{ ATRIBUTO } & \multicolumn{2}{|c|}{ Estudiante } & \multicolumn{2}{|c|}{ Empleados } & \multicolumn{2}{|c|}{ Docentes } & \multicolumn{2}{|c|}{ Directivos } \\
\hline & Kappa & Sig & Kappa & Sig & Kappa & Sig & Kappa & Sig \\
\hline $\begin{array}{l}\text { Cortesía del } \\
\text { personal }\end{array}$ & 0,16 & 0,0 & 0,116 & 0,299 & 0,233 & 0,505 & 0,77 & 0,0 \\
\hline Profesionalismo & 0,122 & 0,0 & 0,168 & 0,158 & 0,22 & 0,001 & 0,0 & 0,0 \\
\hline $\begin{array}{l}\text { Atención } \\
\text { personalizada }\end{array}$ & 0,109 & 0,0 & $-0,93$ & 0,444 & 0,232 & 0,001 & 0,0 & 0,0 \\
\hline $\begin{array}{l}\text { Facilidad en el } \\
\text { acceso al servicio }\end{array}$ & 0,129 & 0,0 & 0,159 & 0,199 & 0,24 & 0,0 & 0,0 & 0,0 \\
\hline $\begin{array}{l}\text { Rapidez en el } \\
\text { servicio }\end{array}$ & 0,107 & 0,0 & 0,68 & 0,541 & 0,221 & 0,001 & 0,77 & 0,45 \\
\hline $\begin{array}{l}\text { Claridad de la } \\
\text { información } \\
\text { recibida }\end{array}$ & 0,110 & 0,0 & 0,65 & 0,604 & 0,65 & 0,304 & $-0,50$ & 0,248 \\
\hline $\begin{array}{l}\text { Cumplimiento del } \\
\text { horario de } \\
\text { servicio }\end{array}$ & 0,16 & 0,0 & $-0,18$ & 0,89 & 0,228 & 0,03 & 1,0 & 0,46 \\
\hline $\begin{array}{l}\text { Instalaciones } \\
\text { físicas }\end{array}$ & 0,147 & 0,0 & 0,145 & 0,226 & 0,176 & 0,003 & 0,6 & 0,7 \\
\hline $\begin{array}{l}\text { Apariencia del } \\
\text { personal }\end{array}$ & 0,17 & 0,0 & $-0,3$ & 0,831 & 0,233 & 0,001 & 0,556 & 0,82 \\
\hline $\begin{array}{l}\text { Limpieza de las } \\
\text { instalaciones }\end{array}$ & 0,18 & 0,0 & 0,108 & 0,397 & 0,115 & 0,105 & 0,50 & 0,248 \\
\hline \begin{tabular}{|l} 
Variedad en \\
servicios \\
médicos \\
\end{tabular} & 0,194 & 0,0 & 0,219 & 0,91 & 0,104 & 0,107 & 0,191 & 0,0 \\
\hline
\end{tabular}

Fuente: Elaboración propia a partir de datos obtenidos 
ISSN: 2602-8085

www.cienciadigital.org

Vol. 3, N³.3, p. 134 - 143, septiembre, 2019

La tabla anterior detalla los valores correspondientes al coeficiente Kappa de Cohen, así como el nivel de significancia, en todos los estratos Sig $<0,05$ en todos los criterios evaluados, lleva a la conclusión de que existe una percepción débil de concordancia entre importancia y satisfacción lo cual es estadísticamente significativa. Esto demuestra las brechas existentes en la gestión de la calidad del servicio prestado por el Centro de atención de salud.

\section{Conclusiones}

- Los resultados de la investigación determinan dimensiones detalladas de los criterios de satisfacción de los usuarios y la evaluación de su importancia desde la perspectiva de los prestadores del servicio. El análisis comparativo de estas dimensiones permite identificar y evaluar las deficiencias actuales en la prestación del servicio de salud y detectar brechas entre el nivel de servicio ofrecido y el nivel percibido y deseado por el usuario.

- Respecto a los factores de mayor importancia considerados por los usuarios se encuentran: Limpieza de las instalaciones, cumplimiento del horario de servicio, profesionalismo, facilidad en el acceso al servicio, cortesía del personal, rapidez en el servicio e instalaciones físicas. Factores que en su conjunto recibieron calificaciones que reflejan bajos niveles de satisfacción en los usuarios.

- Las valoraciones obtenidas tanto de importancia como satisfacción concedidas a los atributos estudiados por parte de los usuarios y prestadores del servicio no presentan coincidencias, situación que se traduce en oportunidades de mejora en la prestación del servicio a partir de las debilidades identificadas.

- Adicional a ello, existen notables diferencias en la valoración del nivel de satisfacción con el servicio entre los estratos que componen el segmento de usuarios. De acuerdo con estos resultados, si bien resulta conveniente investigar la relación entre la importancia y la satisfacción, es preciso realizar un análisis detallado por estrato, identificando aquellos atributos que requieren mayor atención para mejorar la experiencia de todos los usuarios en el centro de salud.

\section{Referencias bibliográficas}

Carlzon, J. (1987). Moments of Truth, Ballinger Pub

Castillo, E. (2005). Escala Multidimensional SERVQUAL. Facultad de Ciencias Empresariales, Universidad del Bío-Bío. Chile.

Curry, A. (2002). Assessing the quality of physiotherapy services using SERVQUAL. International Journal of Health Care Quality Assurance.

Del Cid, A. Méndez, R. y Sandoval, F. (2007). Investigación, Fundamentos y Metodología. Primera Edición. Pearson Educación. México.

Donavedian, A. (1966). Evaluating the quality of medical care. Milbank Memorial Fund Quart.

Duane, D. (2001). Investigación en Administración para la toma de decisiones. Quinta Edición. Thomson Editores S.A. de C.V. México. 
Gallego, J. (2002). Gestión de Alimentos y Bebidas para Hoteles, Bares y Restaurantes (2da. ed.). Editorial Paraninfo, S.A. Madrid, España.

González, J. y Pazmiño, M. (2015). Cálculo e interpretación del Alfa de Cronbach para el caso de validación de la consistencia interna de un cuestionario, con dos posibles escalas tipo Likert. Revista Publicando. 2 (1). p. 62-77.

Hernández, R. Fernández, C. Baptista P. (2006). Metodología de la Investigación. Cuarta Edición. McGraw Hill Interamericana. México.

ISO (2015). ISO 9001: 2015. Sistemas de gestión de calidad - Fundamentos y vocabulario. Organización Internacional de Normalización. Ginebra Suiza.

Koss, E. (1954). The health of regionville. New York: Haffner.

Parasuraman, A.; Zeithmal, V. y Berry, L. (1993). Calidad total en la gestión de servicios. Editorial Díaz de Santos. Madrid.

Rust, I. \& Oliver, R. (1994). Service quality. New directions in theory and practice. California: Sage Publications.

Sainz, J. (2001). La distribución comercial: opciones estratégicas. Madrid Esic.

Thompson, I. (2005). La satisfacción del cliente. [En línea]. Disponible en http//www.pronegocios.net/satisfacciondelcliente/ [Accesado el 2 de mayo de 2019].

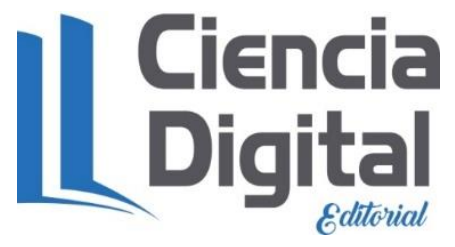




\section{PARA CITAR EL ARTÍCULO INDEXADO.}

Pazmiño Garzón, D., Rodríguez Cotilla, Z., Carrión Torres, M., \& Moncayo Sánchez, Y. (2019). Análisis de la satisfacción en el servicio de salud desde la perspectiva de usuarios y personal de contacto. Ciencia Digital, 3(3.3), 134-143. https://doi.org/10.33262/cienciadigital.v3i3.3.775

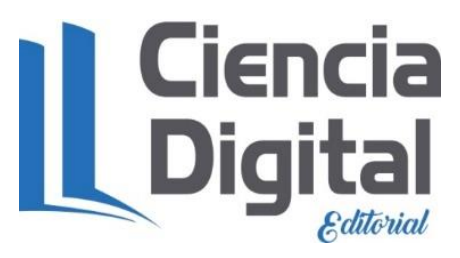

El artículo que se publica es de exclusiva responsabilidad de los autores y no necesariamente reflejan el pensamiento de la Revista Ciencia Digital.

El artículo queda en propiedad de la revista y, por tanto, su publicación parcial y/o total en otro medio tiene que ser autorizado por el director de la Revista Ciencia Digital.
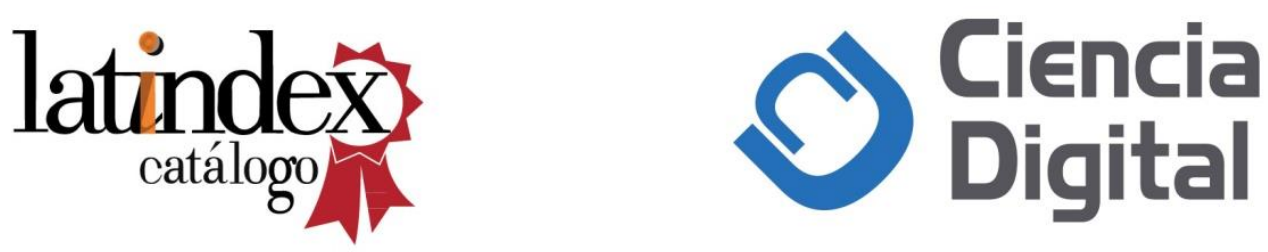